\title{
Dose Validation of Physical Wedged Asymmetric Fields in Artiste Linear Accelerator
}

\author{
Tamer Dawod1, E. M. Abdelrazek², Mostafa Elnaggar³ ${ }^{3}$ Rehab Omar ${ }^{*}$ \\ ${ }^{1}$ Faculty of Medicine, Mansoura University, Mansoura, Egypt \\ ${ }^{2}$ Department of Physics, Faculty of Science, Mansoura University, Mansoura, Egypt \\ ${ }^{3}$ Department of Cancer Management and Research Medical Research Institute, Alexandria University, \\ Alexandria, Egypt \\ ${ }^{4}$ Clinical Oncology and Nuclear Medicine Department, Mansoura University Hospitals, Mansoura, Egypt \\ Email: tamerdawod@gmail.com, emabdelrazek@mans.edu.eg, elnaggarmost@yahoo.co.uk, \\ "ro_elsayed@yahoo.com
}

Received 8 August 2014; revised 5 September 2014; accepted 2 October 2014

Copyright (c) 2014 by authors and Scientific Research Publishing Inc.

This work is licensed under the Creative Commons Attribution International License (CC BY). http://creativecommons.org/licenses/by/4.0/

(c) $\stackrel{\text { (i) }}{\mathrm{EY}}$ Open Access

\begin{abstract}
Aim: The purpose of this study was to make a comparison between measured and calculated physical wedge dose distributions using the superposition algorithm. Settings and Design: The accurate determination of absorbed dose is important radiotherapy because of the relatively steep sigmoidal dose response curves for both tumor control and normal-tissue damage. Materials and Methods: High-energy photons (6 and 10 MV) from Artiste Treatment System Linear Accelerator Machine, available at Alexandria Ayadi Al-Mostakbal Oncology Center, were used. Results and Discussion: The results showed that the difference between measured and calculated wedged isodose curves depends on field size, beam energy, and the angle of the used wedge. Conclusion: The results showed that the presence of a wedge alters the primary and scattered components generated by a linear accelerator and causes beam hardening in 6 and $10 \mathrm{MV}$. The beam hardening increased as the wedge angle increased.
\end{abstract}

\section{Keywords}

Radiotherapy, Wedge, Linear Accelerator, Computerized Treatment Planning System

\section{Introduction}

The accurate determination of absorbed dose is crucial to the success of radiotherapy because of the relatively

"Corresponding author.

How to cite this paper: Dawod, T., Abdelrazek, E.M., Elnaggar, M. and Omar, R. (2014) Dose Validation of Physical Wedged Asymmetric Fields in Artiste Linear Accelerator. International Journal of Medical Physics, Clinical Engineering and Radiation Oncology, 3, 201-209. http://dx.doi.org/10.4236/ijmpcero.2014.34026 
steep sigmoidal dose response curves for both tumor control and normal-tissue damage. A difference of only a few percent in the dose (to the tumor) may separate uncomplicated tumor eradication from either failure to control the tumor through underdosage or serious normal tissue damage through overdosage. There are several papers reporting the results of wedge beam profile measurements from physical, virtual and dynamic filters, using different dosimeters such as diode, ionization chamber, chemical dosimeter, film and numerical methods that use Monte Carlo calculation codes [1]. Irregularly shaped fields have been used for a long time in radiotherapy using photon beams [2]. Radiation therapy treatment planning for many clinical situations requires wedge shaped isodose distributions. In radiation therapy, three different methods are routinely used to produce a wedged dose distribution for a high-energy photon beam. The simplest method is the use of a physical wedge; the second method is the universal wedge technique; the third method is the virtual (dynamic) wedge [3] [4]. The use of mechanical wedge filters is a well-established method for dose inhomogeneity compensation in photon therapy [5], wedge filters, which raise two problems in the radiotherapy practice: progressive attenuation of beam across the field (the thinner side of the wedge attenuates the beam less than the thicker side) and spinning of isodoses curves plate [6] [7].

The physical wedge system requires a separate wedge for each beam width, optimally designed to minimize the loss of beam output [8]. The wedge filter is made by material with high density (e.g. steel or lead), is mounted on a special tray and can be placed at a certain distance from de source in the beam. The wedge filter is characterized by the wedge angle and by the transmission factor [8]-[13]. Physical wedges attenuate beam in both the gradient and nongradient directions of the wedge. For large fields data should also be taken in the nongradient direction to examine the impact of rounding off, due to oblique incidence of the beam and selectively higher attenuation at off axis [14]-[16].

Universal wedge is a single physical wedge $\left(60^{\circ}\right)$ which could generate desired angle $\left(0^{\circ}\right.$ to $\left.60^{\circ}\right)$ with the combination of open and wedged beam [17]. Modern medical accelerators are usually equipped with a dynamic wedge (DW) option. The DW makes use of dynamic movement of one pair of independent jaws on a linac and generates dose distributions equivalent to those produced by a PW placed in static fields [3].

Accurately modeling the distribution of dose in clinical situations is essential to the modern practice of radiotherapy. The introduction of a new dose calculation algorithm into a commercial TPS warrants extensive validation by the medical physics community before the algorithm is accepted for clinical implementation. Thus there is an impetus to critically examine the performance of the superposition dose deposition method. Selecting a proper set of validation tests to be applied and identifying appropriate criteria upon which to judge the results are essential to the evaluation process [18].

The Xio superposition dose deposition method is an adaptation of the "collapsed cone" dose calculation method [19]. As with FFT Convolution, all superposition calculations are done in beam coordinates, and the dose in the beam coordinates is interpolated to the user specified calculation volume. This study is aimed to validate the wedged asymmetrical fields by comparing between measured and calculated physical wedge dose distributions using the Xio superposition algorithm.

\section{Materials and Methods}

\subsection{Beam Set Arrangements}

High-energy photons (6 and $10 \mathrm{MV}$ ) from Artiste Treatment System Linear Accelerator machine, available at Alexandria Ayadi Al-Mostakbal Oncology Center, were used. A linear accelerator having Physical wedge angles of $15^{\circ}, 30^{\circ}, 45^{\circ}$ and $60^{\circ}$ was used to produce 6 and $10 \mathrm{MV}$ photon beams. The dose distributions were calculated by CMS (Xio 4.5) 3D planning system 3DTPS. PWs with asymmetric fields for $10 \mathrm{~cm} \times 10 \mathrm{~cm}$, (X1 = 5, $\mathrm{X} 2=5 \& \mathrm{Y} 1=2.5, \mathrm{Y} 2=7.5),(\mathrm{X} 1=5, \mathrm{X} 2=5 \& \mathrm{Y} 1=5, \mathrm{Y} 2=5),(\mathrm{X} 1=5, \mathrm{X} 2=5 \& \mathrm{Y} 1=7.5, \mathrm{Y} 2=2.5)$ at depths of $d_{\max }, 5,10,20 \mathrm{~cm}$ with source to surface distance (SSD) of $100 \mathrm{~cm}$ as shown in Figure 1. Calculations were performed in a phantomcreated by the Xio 3DTPS with a homogeneous density of $1 \mathrm{~g} / \mathrm{cm}^{3}$. The dose was calculated for all PW angles for 6 and 10 MV photon beams at each depth.

Dose profile curves were measured at predefined depths in water phantom with a PTW dosimetry system with with two semiflex $(0.125 \mathrm{cc})$ ionization chamber. The position of the ionization chamber is critical in the case of wedged beams due to the dose gradient in the direction of the wedge. The chamber was mounted in a holder, placed in a $50 \mathrm{~cm} \times 50 \mathrm{~cm} \times 50 \mathrm{~cm}$ PTW three dimensional water phantom. The water surface was leveled at SSD of $100 \mathrm{~cm}$. The gantry of the treatment unit was set to $0^{\circ}$. The linac was set to deliver 200 monitor units 


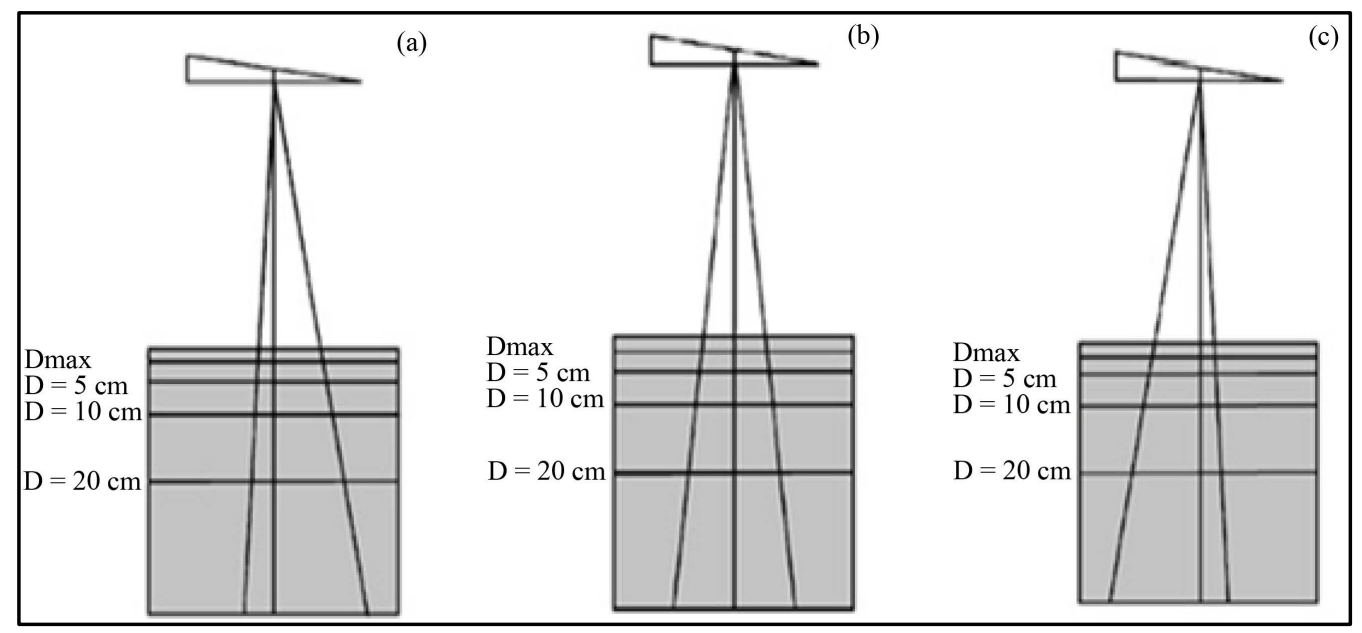

Figure 1. View of the beam setup showing the depths of measurement (a) in the asymmetrical setting (X1 $=5, \mathrm{X} 2=5, \mathrm{Y} 1=2.5, \mathrm{Y} 2=7.5)$, (b) in the symmetrical setting $(\mathrm{X} 1=5, \mathrm{X} 2=5, \mathrm{Y} 1=5, \mathrm{Y} 2=5)$, and (c) in the asymmetrical setting $(\mathrm{X} 1=5, \mathrm{X} 2=5, \mathrm{Y} 1=7.5, \mathrm{Y} 2=2.5)$.

(MUs) per minute. To reduce the variability of working conditions, the dosimetry measurements were perfo-r med in a single session. The measured isodose was compared with the calculated isodose obtained from the XiO 3DTPS by using PTW VeriSoft software (Version 4).

\subsection{Calculated Dose Validation}

In order to compare calculated and measured doses, we used PTW VeriSoft software (Version 4) to verify the treatment plan by comparing calculated data to its corresponding measured in phantom.

According to the tolerance values for homogeneous simple fields, the penumbra region should be within 2 $\mathrm{mm}$ or $10 \%$. By just studying the profiles by eye it is hard to say, especially in the z-direction in the penumbra region, if the result is within the tolerance. A gamma evaluation with $3 \%$ and $3 \mathrm{~mm}$ criteria, revealing that it is only in the penumbra region that acceptance fails. The colours of the palette range are set to be green for $100 \%$ $(\gamma=1)$, and accepted regions are green and most yellow. Regions that fail are shown in red. The gamma evaluation method is not a good tool for evaluation of low dose regions, where the calculation can fail though it is within the set criteria. For example if we are comparing two dose points of $4 \%$ and $1 \%$ dose, and the dose criteria is set to be $2 \%$, this will lead to a gamma value larger than $1((4 \%-1 \%) / 2 \%)$. The $3 \%$ dose difference can still be within acceptable tolerances but the gamma calculation fails.

The difference matrix of two detector array matrices is determined by comparison of the measured and the calculated asymmetric field size are expressed as a percentage of the locally measured dose by using the following equation

$$
\text { Diffrence }(\%)=((\text { Dcalculated }- \text { Dmeasured }) / \text { Dmeasured }) \times 100
$$

\section{Results}

A physical wedge is an angled piece of lead or steel that is placed through the beam path to produce a gradient in radiation intensity. Manual intervention is required to place physical wedges on the treatment unit's collimator assembly [6].

The measured data were compared with data from treatment planning system. The statistical analysis was performed using the PTW-VERISOFT program to evaluate the differences between the data.

Twenty four asymmetric fields with 6 and 10 MV energy were used for comparison and verification. Figure 1 shows the view of the beam setup showing the field sizes of measurement in the asymmetrical setting $(\mathrm{X} 1=5$, $\mathrm{X} 2=5, \mathrm{Y} 1=2.5, \mathrm{Y} 2=7.5), \mathrm{b})$ in the symmetrical setting $(\mathrm{X} 1=5, \mathrm{X} 2=5, \mathrm{Y} 1=5, \mathrm{Y} 2=5)$, and $\mathrm{c})$ in the asymmetrical setting $(\mathrm{X} 1=5, \mathrm{X} 2=5, \mathrm{Y} 1=7.5, \mathrm{Y} 2=2.5)$.

Table 1 and Table 2 represent the difference between measured dose and calculated dose at different isodose 
Table 1. Difference between measured dose and calculated dose at different isodose lines for symmetric and asymmetric fields for the $6 \mathrm{MV}$ and the four physical wedge angles.

\begin{tabular}{|c|c|c|c|c|}
\hline \multicolumn{5}{|c|}{ GAMMA INDEX (3 mm) } \\
\hline \multicolumn{5}{|c|}{ Y-dir, $6 \mathrm{MV}$} \\
\hline \multicolumn{5}{|c|}{ Wedge 15} \\
\hline F.S $(\mathrm{cm})$ & Evaluated Dose Points & Passed & Failed & Result \\
\hline$(-2.5,7.5)$ & & $96.00 \%$ & $4.00 \%$ & $96.00 \%$ \\
\hline$(-5,5)$ & $100 \%$ & $98.20 \%$ & $1.80 \%$ & $98.20 \%$ \\
\hline$(-7.5,2.5)$ & & $97.40 \%$ & $3 \%$ & $97.40 \%$ \\
\hline \multicolumn{5}{|c|}{ Wedge 30} \\
\hline$(-2.5,7.5)$ & & $88.50 \%$ & $11.50 \%$ & $88.50 \%$ \\
\hline$(-5,5)$ & $100 \%$ & $98.40 \%$ & $1.60 \%$ & $98.40 \%$ \\
\hline$(-7.5,2.5)$ & & $95.70 \%$ & $4.30 \%$ & $95.70 \%$ \\
\hline \multicolumn{5}{|c|}{ Wedge 45} \\
\hline$(-2.5,7.5)$ & & $85.30 \%$ & $14.70 \%$ & $85.30 \%$ \\
\hline$(-5,5)$ & $100 \%$ & $97.10 \%$ & $2.90 \%$ & $97.10 \%$ \\
\hline$(-7.5,2.5$ & & $97.00 \%$ & $3 \%$ & $97.00 \%$ \\
\hline \multicolumn{5}{|c|}{ Wedge 60} \\
\hline$(-2.5,7.5)$ & & $93.10 \%$ & $6.90 \%$ & $93.10 \%$ \\
\hline$(-5,5)$ & $100 \%$ & $95.90 \%$ & $4.10 \%$ & $95.90 \%$ \\
\hline$(-7.5,2.5)$ & & $98.10 \%$ & $1.90 \%$ & $98.10 \%$ \\
\hline
\end{tabular}

Table 2. Difference between measured dose and calculated dose at different isodose lines for symmetric and asymmetric fields for the $10 \mathrm{MV}$ and the four physical wedge angles.

\begin{tabular}{|c|c|c|c|c|}
\hline \multicolumn{5}{|c|}{ GAMMA INDEX (3 mm) } \\
\hline \multicolumn{5}{|c|}{ Y-dir. $10 \mathrm{MV}$} \\
\hline \multicolumn{5}{|c|}{ Wedge 15} \\
\hline F.S (cm) & Evaluated Dose Points & Passed & Failed & Result \\
\hline$(-2.5,7.5)$ & & $97.00 \%$ & $3.00 \%$ & $97.00 \%$ \\
\hline$(-5,5)$ & $100 \%$ & $98.40 \%$ & $1.60 \%$ & $98.40 \%$ \\
\hline$(-7.5,2.5$ & & $98.00 \%$ & $2 \%$ & $98.00 \%$ \\
\hline \multicolumn{5}{|c|}{ Wedge 30} \\
\hline$(-2.5,7.5)$ & & $94.8 \%$ & $5.2 \%$ & $94.8 \%$ \\
\hline$(-5,5)$ & $100 \%$ & $97.40 \%$ & $2.60 \%$ & $97.40 \%$ \\
\hline$(-7.5,2.5)$ & & $98.40 \%$ & $1.60 \%$ & $98.40 \%$ \\
\hline \multicolumn{5}{|c|}{ Wedge 45} \\
\hline$(-2.5,7.5)$ & & $94.20 \%$ & $5.80 \%$ & $94.20 \%$ \\
\hline$(-5,5)$ & $100 \%$ & $96.30 \%$ & $3.70 \%$ & $96.30 \%$ \\
\hline$(-7.5,2.5$ & & $97.10 \%$ & $3 \%$ & $97.10 \%$ \\
\hline \multicolumn{5}{|c|}{ Wedge 60} \\
\hline$(-2.5,7.5)$ & & $90.00 \%$ & $10.00 \%$ & $90.00 \%$ \\
\hline$(-5,5)$ & $100 \%$ & $97.30 \%$ & $2.70 \%$ & $97.30 \%$ \\
\hline$(-7.5,2.5)$ & & $97.00 \%$ & $3.00 \%$ & $97.00 \%$ \\
\hline
\end{tabular}


lines for symmetric and asymmetric fields for the dual energy and the four physical wedge angles. Figure 2 and Figure 3 indicated comparison of the dose distribution by the gamma method for energies, different field size, and different wedge angles. The result of the comparison was displayed for both images using the gamma method. It defines a percentage difference between the measured and the calculated dose at certain distance. For example, for gamma factor 3 we can say that $100 \%$ corresponds to $3 \%$ of the difference between the measured and the calculated dose within a distance of $3 \mathrm{~mm}$. The obtained image is colour scaled. In Figure 2 and Figure 3 , the green surfaces represent an area where the difference between the doses is lower than $3 \%$; the red areas exhibit the dose differences equal or higher than $3 \%$.

From all obtained gamma indexes for different wedge angles and different field sizes we can say, that the differences between the measured and the calculated distributions for most isodoses have not exceeded $5 \%$ within the distance of $3 \mathrm{~mm}$. For the symmetric field, most calculated results match the measurement within $3 \%$, only for some cases the difference exceed 3\% for (wedge 60, $6 \mathrm{MV}$ ) and (wedge 45, $10 \mathrm{MV}$ ).

For the asymmetric field, most calculated results match the measurement within $6 \%$. For asymmetric field size ( $\mathrm{X}=10 \& \mathrm{Y} 1=2.5, \mathrm{Y} 2=7.5$ ), the difference between measured and calculated up to $14 \%$ for $6 \mathrm{MV}$, and up to $10 \%$ for $10 \mathrm{MV}$ at the thin edge of the wedge direction . In general, for cases where the more asymmetric field is toward the thick edge of the wedge, it leads to the greatest difference between the measured and calculated doses. The doses under the thin edge of the wedges are usually underestimated while the opposite occurs for the thick end of the wedge where the doses are overestimated in most cases. The wedge angle changes very slowly with field size, but appreciably with depth, depending on the photon energy.

In general, the wedge filter alters the beam quality by preferentially attenuating the lower energy photons

\section{1- W 15, 6MV}
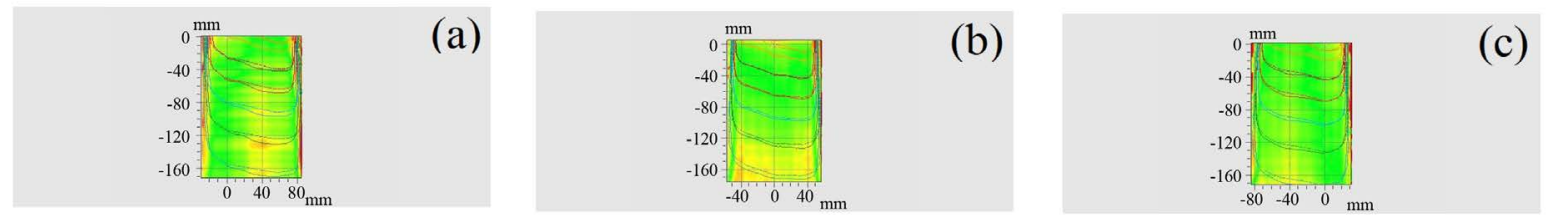

\section{$2-W 30,6 M V$}
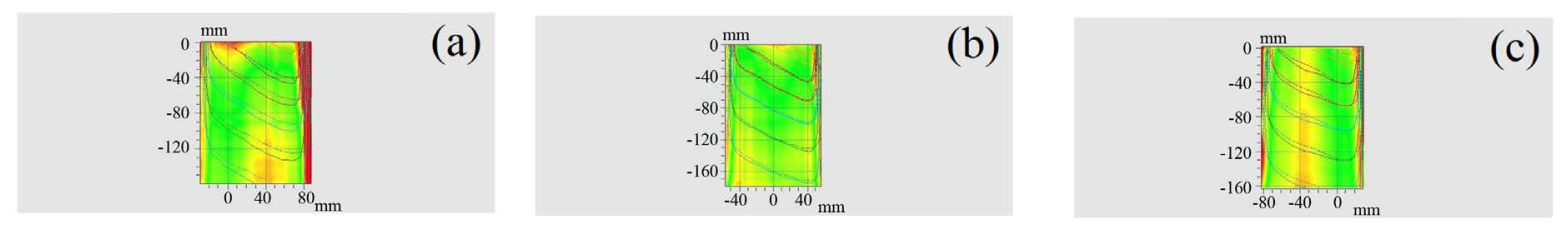

3- W $45,6 \mathrm{MV}$
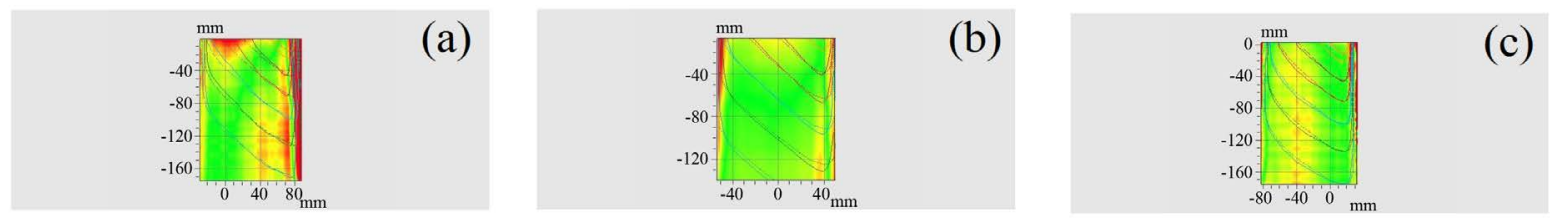

\section{4- $\mathrm{W} 60,6 \mathrm{MV}$}

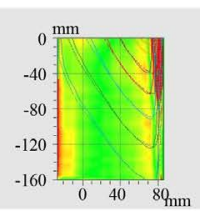

(a)
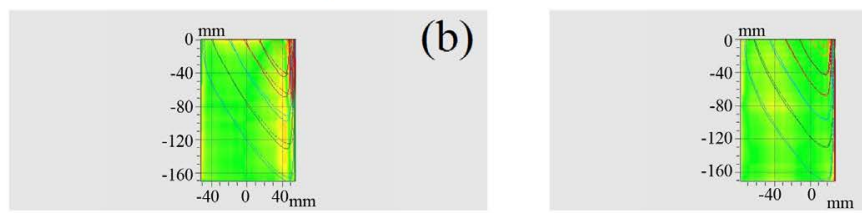

(c)

Figure 2. Gamma distribution for different field using the superposition algorithm for 15, 30, 45 degrees and 60 degrees physical wedge (PW), for $6 \mathrm{MV}$. The upper parts of the distributions represent the low-dose region of the wedged field; green indicates regions where gamma $\leq 1$; and red indicates gamma $>1$ (individual criteria: $\Delta \% \mathrm{D}=3 \%, \mathrm{DTA}=3 \mathrm{~mm}$ ). 


\section{1- W15, 10MV}
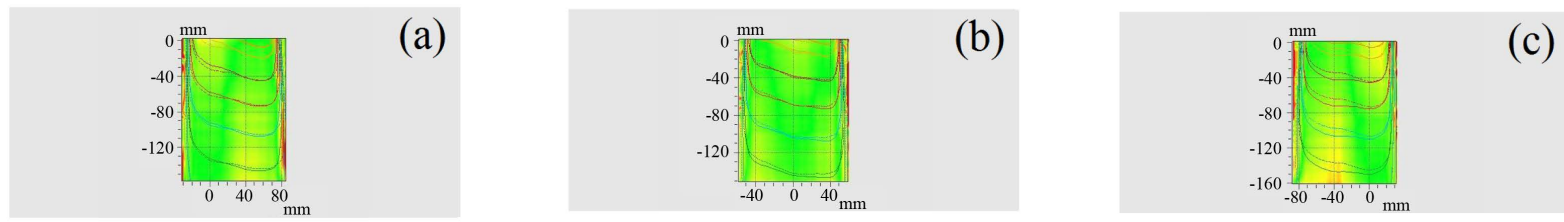

\section{2- W30, 10MV}
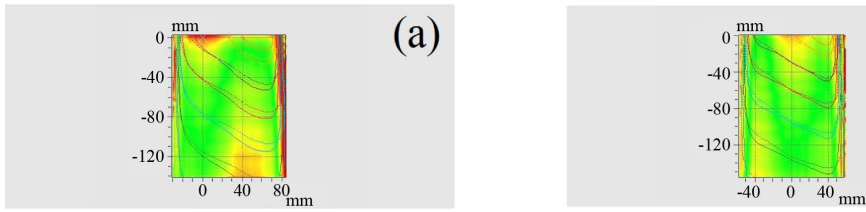

(b)

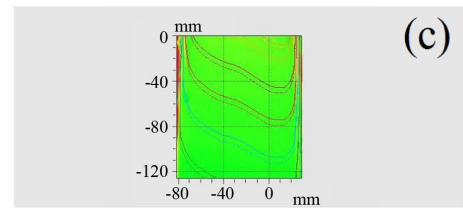

\section{3- W 45, 10MV}

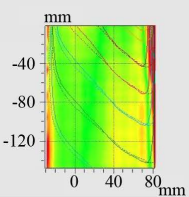

(a)

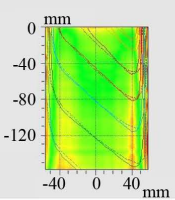

(b)

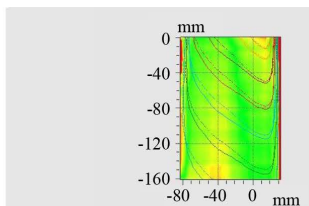

(c)

\section{4- W $60,10 \mathrm{MV}$}

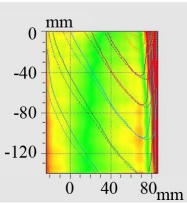

(a)

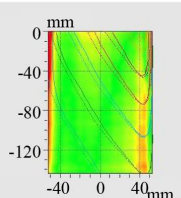

(b)

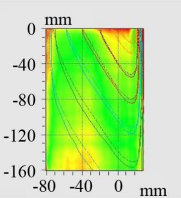

(c)

Figure 3. Gamma distribution for different field using the superposition algorithm for 15, 30, 45 degrees and 60 degrees physical wedge $(\mathrm{PW})$, for $6 \mathrm{MV}$. The upper parts of the distributions represent the low-dose region of the wedged field; green indicates regions where gamma $\leq 1$; and red indicates gamma $>1$ (individual criteria: $\Delta \% \mathrm{D}=3 \%, \mathrm{DTA}=3 \mathrm{~mm}$ ).

(beam hardening) and, to a lesser extent, by Compton scattering, which results in energy degradation (beam softening). For x-rays, there can be some beam hardening, and consequently, the depth dose distribution can be somewhat altered, especially at large depths [8] [20]-[21]. When a field is collimated asymmetrically, one needs to take into account changes in the collimator scatter, phantom scatter, and off-axis beam quality. The latter effect arises as a consequence of using beam-flattening filters (thicker in the middle and thinner in the periphery), which results in greater beam hardening close to the central axis compared with the periphery of the beam [8].

The noticeable increase in the PDD values at all depths in phantom can be attributed to two factors: 1) differences in scatter produced from the thin and thick sides of the wedge filter and 2) beam hardening effects. If the amount of scatter produced on one side of the wedge filter increases more than it is reduced on the other side, as is the case with sigmoidal shaped wedges, a larger portion of scatter will be measured on the beam central axis. This increase in scatter generated from the wedge filter, combined with an increasing amount of phantom scatter with depth, yields a higher percentage depth dose compared with the open field photon beam [22]-[23].

\section{Discussion}

Published data for the TPS dose calculations present significant variation. The first criteria published by Van Dyk et al. in 1993 [23] are characterized by increased tolerance limits due to the fact that most of the TPS were using two-dimensional algorithms at the time. The recommendations of AAPM TG53 report in 1998 by Fraass 
et al. [24], and Venselaar and Welleweerd in 2001 [25] recently showed for a number of commercial treatment planning systems, that the algorithms for calculating monitor units for wedged asymmetric have their limitation. Deviation up to $13 \%$ between measured and calculated dose were observed under the thick and the thin end of the wedge are generally more strict, but realistic for a properly functioning dose calculation algorithm. When the complexity of the geometry increases, however, tolerance limits may have to be less strict relative to beam modeling geometry [3].

The highest difference of our results is higher than Venselaar and Welleweerd and lower than Caprile et al. [26] which riches to $28.5 \%$ for pencil-beam convolution (PBC) for field size $20 \times 20$. The disagreement regions correspond to the edge of the field where the penumbra is not well modeled.

The results showed that the comparison between the measured and calculated physical wedge depends strongly on field size, increasing as field size decreases, the results indicated also that the quality of the radiation beam plays a significant role in the calculation of the PW. With every changing wedge angle, the hardening and softening of the beam varies, indicating the vital role of the wedge factor dependence of the dose. Thus, the quality of the beam itself is of significant importance in the dose precision. This result agreed with Muhammad Maqbool et al. [27]. As the wedge angle increased, the difference increased, this agreed with Ravinder Nath et al., M. Pasquino, et al. and M. Momennezhad, et al. [28]-[30].

\section{Conclusion}

Our results have confirmed the conformity of the treatment at Ayadi Al-Mostakbal Oncology Center when PW is used. In this study, we describe in details only one step of the verification. Within the process of implementation of PW, every workstation has to verify also the stability of PW, the PW comparison between the measured and the calculated isodoses of all PW. This is a subject of the ongoing works. The results showed that the presence of a wedge alters the primary and scattered components generated by a linear accelerator and causes beam hardening in 6 and $10 \mathrm{MV}$. The beam hardening increased as the wedge angle increased.

\section{References}

[1] de Oliveira, L.N., Carmen, S.G.C., Fernanda, C. and de Adelaide, A. and de Almeida, C.E. (2009) 6 MV Wedge Photon Beam Profiles with the Fricke Xylenol Gel Dosimeter. Brazilian Journal of Physics, 39, 615-618. http://dx.doi.org/10.1590/S0103-97332009000600001

[2] Storchi, P. and Woudstra, E. (1996) Calculation of the Absorbed Dose Distribution Due to Irregularly Shaped Photon Beams Using Pencil Beam Kernels Derived from Basic Beam Data. Physics in Medicine and Biology, 41, 637-656. http://dx.doi.org/10.1088/0031-9155/41/4/005

[3] Anjum, M.N., Qadir, A. and Afzal, M. (2008) Dosimetric Evaluation of a Treatment Planning System Using Pencil Beam Convolution Algorithm for Enhanced Dynamic Wedges with Symmetric and Asymmetric Fields. International Journal of Radiation Research, 5, 169-174.

[4] Dai, J., Zhu, Y. and Wu, X. (2001) Verification of the super omni wedge concept. Physics in Medicine and Biology, 46, 2447-2455. http://dx.doi.org/10.1088/0031-9155/46/9/313

[5] Huntzinger, C.J. (1992) Dynamic Wedge: A Physicist’s Perspective. Varian Dynamic Wedge Users' Meeting Proceedings, Calagary, August 1992, 24.

[6] Strunga, E. (1995) Physical Parameters for Wedge Filter Used in Radiotherapy. Radiotherapy and Oncology, 37, 59-59. http://dx.doi.org/10.1016/0167-8140(96)80663-3

[7] Dumitru, M., Rebegea, L., Praisler, M., Strunga, E. and Dumitrache, M. (2013) Issues in Radiotherapy Practice Due the Presence of Wedge Filters. Romanian Reports in Physics, 65, 456-467.

[8] Khan, F.M. (1994) The Physics of Radiation Therapy. 2nd Edition, Williams and Wilkins, London.

[9] Leavitt, D.D., Martin, M., Moeller, J.H. and Lee, W.L. (1990) Dynamic Wedge Field Techniques through ComputerControlled Collimator Motion and Dose Delivery. Medical Physics, 17, 87-91. http://dx.doi.org/10.1118/1.596533

[10] Klein, E.E., Low, D.A., Meigooni, A.S. and Purdy, J.A. (1995) Dosimetry and Clinical Implementation of Dynamic Wedge. International Journal of Radiation Oncology*Biology*Physics, 31, 583-592. http://dx.doi.org/10.1016/0360-3016(94)00369-V

[11] Leavitt, D.D. and Klein, E. (1997) Dosimetry Measurement Tools for Commissioning Enhanced Dynamic Wedge. Medical Dosimetry, 22, 171-176. http://dx.doi.org/10.1016/S0958-3947(97)00014-9

[12] Gibbons, J.P. (1998) Calculation of Enhanced Dynamic Wedge Factors for Symmetric and Asymmetric Photon Fields. 
Medical Physics, 25, 1411-1418. http://dx.doi.org/10.1118/1.598313

[13] Prabhakar, R., Julka, P.K. and Rath, G.K. (2008) Can Field in Field Technique Replace Wedge Filter in Radiotherapy Treatment Planning: A Comparative Analysis in Various Treatment Sites. Australasian Physics \& Engineering Sciences in Medicine, 31, 317-324. http://dx.doi.org/10.1007/BF03178601

[14] Das, I.J., Cheng, C.-W., Watts, R.J., Ahnesjö, A., Gibbons, J., Li, X.A., et al. (2008) Accelerator Beam Data Commissioning Equipment and Procedures: Report of the TG-106 of the Therapy Physics Committee of the AAPM. Medical Physics, 35, 4186.

[15] Keall, P., Zavgorodni, S., Schmidt, L. and Haskard, D. (1997) Improving Wedged Field Dose Distributions. Physics in Medicine and Biology, 42, 2183-2192. http://dx.doi.org/10.1088/0031-9155/42/11/013

[16] Myler, U. and Szabo, J.J. (2002) Dose Calculation along the Nonwedged Direction. Medical Physics, 29, 746-754.

[17] Breitman, K., Rathee, S., Newcomb, C., Murray, B., Robinson, D., Field, C., et al. (2007) Experimental Validation of the Eclipse AAA Algorithm. Journal of Applied Clinical Medical Physics, 8, 76-92.

[18] Miften, M.M., Beavis, A.W. and Marks, L.B. (2002) The Influence of the Dose Calculation Model on Treatment Plan Evaluation in Conformal Therapy: A Three Case Study. Medical Dosimetry, 27, 51-57. http://dx.doi.org/10.1016/S0958-3947(02)00088-2

[19] Parker, W. and Patrocinio, H. (2005) Clinical Treatment Planning in External Photon Beam Radiotherapy. In: Podgorsak, E.B., Ed., Radiation Oncology Physics: A Handbook for Teachers and Students, International Atomic Energy Agency, Vienna, 219.

[20] Mayles, P., Nahum, A. and Rosenwald, J.C. (2007) Handbook of Radiotherapy Physics: Theory and Practice. Taylor \& Francis Group, LLC, London. http://dx.doi.org/10.1201/9781420012026

[21] Knöös, T. and Wittgren, L. (1991) Which Depth Dose Data Should Be Used for Dose Planning When Wedge Filters Are Used to Modify the Photon Beam? Physics in Medicine and Biology, 36, 255-266. http://dx.doi.org/10.1088/0031-9155/36/2/009

[22] Podgorsak, M.B., Kubsad, S.S. and Paliwal, B.R. (1993) Dosimetry of Large Wedged High Energy Photon Beams. Medical Physics, 20, 369-372. http://dx.doi.org/10.1118/1.597078

[23] Van Dyk, J., Barnett, R., Cygler, J. and Shragge, P. (1993) Commissioning and Quality Assurance of Treatment Planning Computers. International Journal of Radiation Oncology*Biology*Physics, 26, 261-273. http://dx.doi.org/10.1016/0360-3016(93)90206-B

[24] Fraass, B., Doppke, K., Hunt, M., Kutcher, G., Starkschall, G., Stern, R., et al. (1998) American Association of Physicists in Medicine Radiation Therapy Committee Task Group 53: Quality Assurance for Clinical Radiotherapy Treatment Planning. Medical Physics, 25, 1773. http://dx.doi.org/10.1118/1.598373

[25] Venselaar, J. and Welleweerd, H. (2001) Verification of Dose Calculations with a Treatment Planning System for Open and Wedged Fields of a Cobalt-60 Unit with a New Asymmetric Collimator. Medical Dosimetry, 26, 309-314. http://dx.doi.org/10.1016/S0958-3947(01)00081-4

[26] Caprile, P.F., Venencia, C.D. and Besa, P. (2007) Comparison between Measured and Calculated Dynamic Wedge Dose Distributions Using the Anisotropic Analytic Algorithm and Pencil Beam Convolution. Journal of Applied Clinical Medical Physics, 8, 47-54.

[27] Maqbool, M., Muhammad, W., Shahid, M., Ahmad, M. and Matiullah, M. (2010) Accuracy Checks of Physical Beam Modifier Factors Algorithm Used in Computerized Treatment Planning System for a 15 MV Photon Beam. Reports of Practical Oncology and Radiotherapy, 14, 214-220.

[28] Nath, R., et al. (1994) AAPM Code of Practice for Radiotherapy Accelerators. AAPM Report No. 47, American Institute of Physics, Woodbury.

[29] Pasquino, M., Casanova Borca, V., Tofani, S. and Ozzello, F. (2009) Verification of Varian Enhanced Dynamic Wedge Implementation in Masterplan Treatment Planning System. Journal of Applied Clinical Medical Physics, 2, 2867.

[30] Momennezhad, M., Bahreyni Toosi, M.T., Sadeghi, R., Gholamhoseinian, H. and Nasseri, Sh. (2010) A Monte Carlo Simulation and Dosimetric Verification of Physical Wedges Used in Radiation Therapy. International Journal of Radiation Research, 7, 223-227. 


\section{Abbreviations and Acronyms}

cc: Cubic Centimeter

cm: Centimeter

dir: Direction

FFT: Fast Fourier Transform

F.S.: Field Size

MUs: Monitor Units

MV: Mega Voltage

PW: Physical Wedge

TPS: Treatment Planning System

XiO: Name of three dimensions treatment planning system 
Scientific Research Publishing (SCIRP) is one of the largest Open Access journal publishers. It is currently publishing more than 200 open access, online, peer-reviewed journals covering a wide range of academic disciplines. SCIRP serves the worldwide academic communities and contributes to the progress and application of science with its publication.

Other selected journals from SCIRP are listed as below. Submit your manuscript to us via either submit@scirp.org or Online Submission Portal.
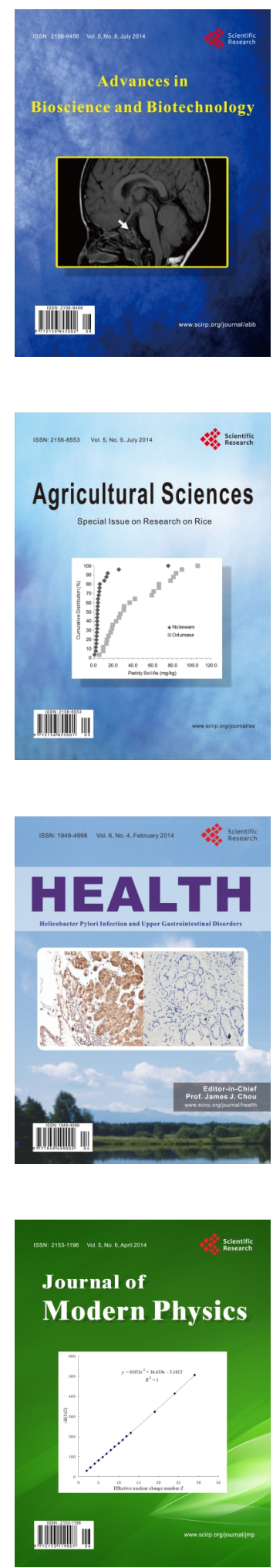
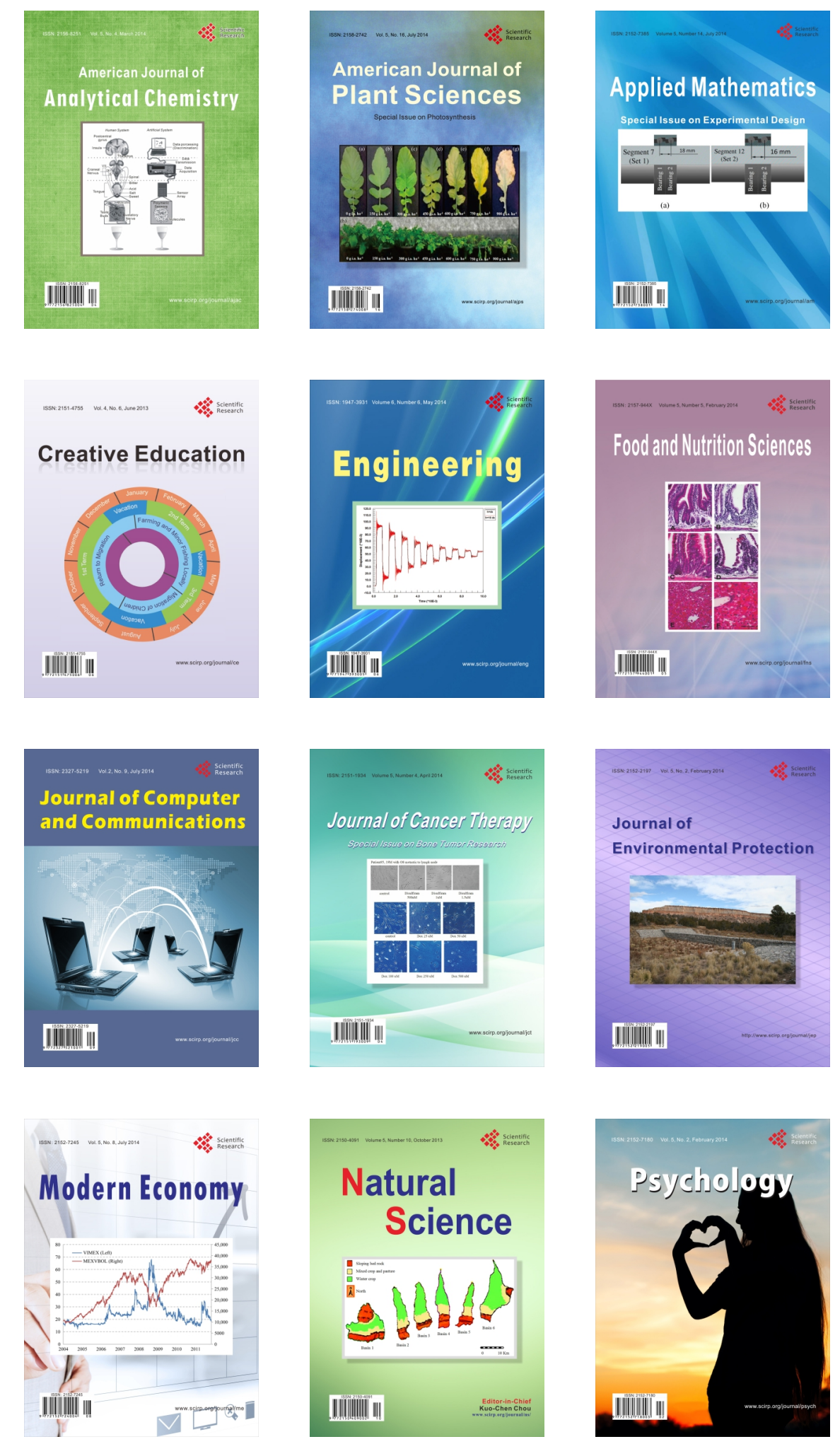